\title{
Development of the Waseda CALET Operations Center (WCOC) for Scientific Operations of CALET
}

\author{
Yoichi Asaoka* for the CALET collaboration \\ Research Institute for Science and Engineering, Waseda University (JP) \\ E-mail: voichi.asaokadaoni.waseda.jp
}

The CALET project aims at a long duration observation of high energy cosmic rays onboard the International Space Station (ISS). The CALET detector features a very thick calorimeter of 30 radiation-lengths which consists of imaging and total absorption calorimeters. It will directly measure the cosmic-ray electron spectrum in the energy range of $1 \mathrm{GeV}-20 \mathrm{TeV}$ with $2 \%$ energy resolution. The data obtained with CALET onboard ISS will be transferred to JAXA using two data relay satellite systems operated by NASA and JAXA, respectively. To operate the CALET onboard ISS, the CALET Ground Support Equipment (CALET-GSE) is being prepared in JAXA. Simultaneously, Waseda CALET Operations Center (WCOC) is being established to perform operations and monitoring related to the scientific mission. The real-time data received by CALET-GSE is immediately transferred to WCOC. Scientific raw data are also transferred to WCOC on an hourly basis after time-order correcting and complementing replay data. Mission operations at WCOC includes the following roles:

1. Real-time monitoring and operations: To monitor the CALET observation and its status in real-time, Quick Look (QL) programs are developed to visualize and summarize both cosmic-ray event data and housekeeping data. Operators at WCOC use QLs to understand the CALET situation and validate the observation on orbit in 24 hour. Upon necessity, appropriate commands will be issued through CALET-GSE.

2. Operation planning: Scheduled command sequences are utilized to control the CALET observation mode on orbit. Calibration data taking such as pedestal and penetrating particle events, low energy electron data taking at high geomagnetic latitude, and other dedicated trigger modes can be scheduled along with the ISS orbit.

3. Scientific data processing: The scientific raw data called CALET Level-0 data are processed to CALET Level-1 data, and Level-1 data are distributed to the collaboration for scientific analysis. Quick analyses based on both Level-0 and Level-1 data are performed and their results are used to feedback for better operation planning and realtime monitoring.

In this contribution, we will review the role of WCOC and report WCOC development.

The 34th International Cosmic Ray Conference,

30 July- 6 August, 2015

The Hague, The Netherlands

\footnotetext{
* Speaker.
} 


\section{Introduction}

The CALorimetric Electron Telescope (CALET) project [四] primarily aims at discovery of nearby cosmic-ray accelerators and search for dark matter by precisely measuring electron and gamma-ray spectra in a wide energy range from $\mathrm{GeV}$ to $\mathrm{TeV}$. It installs a high performance particle detector equipped with thick large-area calorimeter on International Space Station (ISS). CALET will perform a long term observation for two years extensible to 5 years or more, following a launch to ISS scheduled on August 2015.

CALET features very-thick calorimeter which consists of imaging and total absorption calorimeters (See Fig. (). The calorimeter of 30 radiation-length thickness completely absorbs electron shower energy in TeV energy range and identifies electrons from overwhelming protons using the difference in shower development in the fullyactive and thick calorimeter. Longterm observation with large-area detector is granted by observation onboard ISS. By combining all of these features, it becomes possible to precisely measure the total electron spectrum up to $20 \mathrm{TeV}$ for the first time. Main components of cosmic rays such as protons, heliums and heavier nuclei can be measured up to PeV. Including electrons and gamma-rays, it is expected to make unique observations by extending the previous limits of direct measurements.

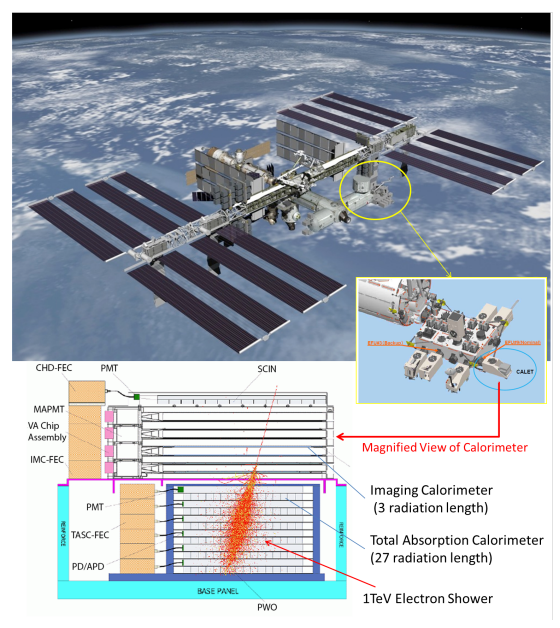

Figure 1: CALET detector onboard ISS on the Japanese Experiment Module - Exposed Facility [四].

As shown in the left panel of Fig. 凹, the data obtained with CALET onboard ISS will be transferred to JAXA. To operate and to monitor the CALET onboard ISS, the CALET Ground Support Equipment (CALET-GSE) at JAXA and Waseda CALET Operations Center (WCOC) in Waseda Univ. are established. The real-time data received by CALET-GSE are immediately transferred to WCOC. Scientific raw data are also transferred to WCOC on an hourly basis after time-order correcting and complementing replay data at JAXA. For the CALET project which aims at unique scientific goals by challenging the boundary of statistics and accuracy, it is very important to maintain detector performance and to carry out observation with high efficiency during the long term operations aboard ISS. WCOC is responsible for this in the CALET operations. Mission

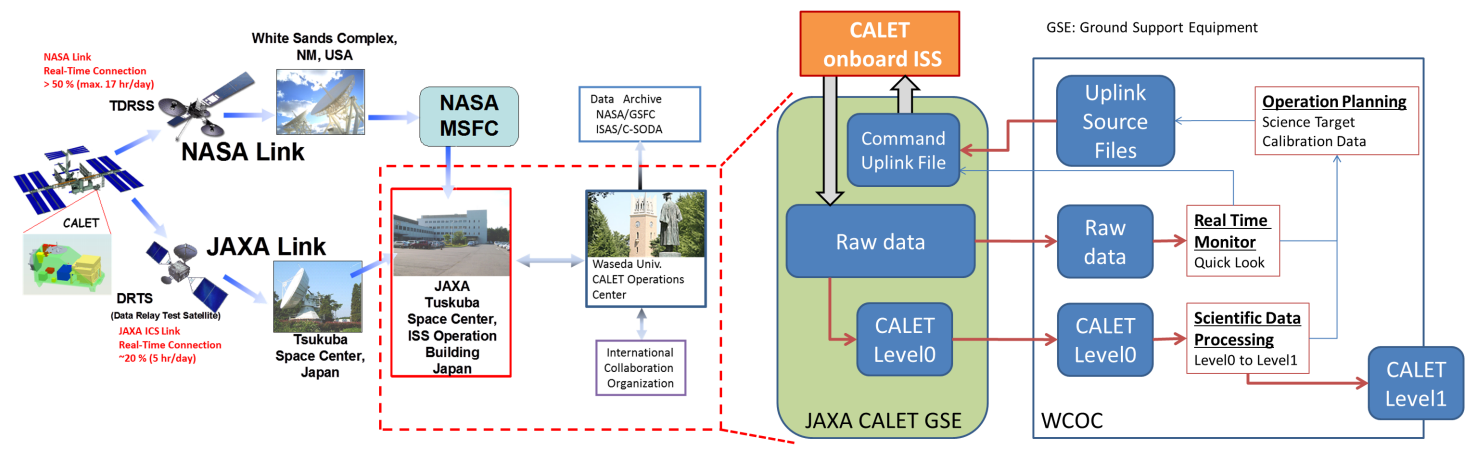

Figure 2: CALET data flow from orbit to ground. In the right side, the dataflow of the CALET ground system is summarized. Interfaces to JAXA CALET-GSE corresponding to each role of WCOC are defined. 
operations at WCOC includes (1) real-time monitoring and operations, (2) operations planning, and (3) processing raw, Level-0, scientific data to Level-1 data that will be used for scientific analysis. As shown in the right panel of Fig. \, there is an interface to JAXA ground system corresponding to each role of WCOC, making it possible to communicate with CALET onboard ISS.

The paper is organized as follows. In Section 2 the CALET detector system including trigger and data acquisition is briefly summarized. In Section 3, the CALET telemetry data simulation system which is used to develop WCOC is described. In Section 4 roles of WCOC is explained in detail. And lastly, the summary and conclusion is given in Section 5.

\section{The CALET Detector System}

\subsection{Detector Components}

The CALET detector (See Fig. $\mathbb{W}$ ) consists of Charge Detector (CHD) which identifies the charge of incident particle, IMaging Calorimeter (IMC) which reconstructs the track of incident particle and images the initial shower development, and Total AbSorption Calorimeter (TASC) which absorbs the whole energy of incoming particle and identifies the particle species by utilizing the segmented scintillator arrays. Combining these components, the CALET detector features (1) proton rejection factor of more than $10^{5}$, (2) $2 \%$ energy resolution, (3) $0.1-0.5^{\circ}$ angular resolution, and (4) large geometrical factor of order of $0.1 \mathrm{~m}^{2} \mathrm{sr}$. The sufficiently high rejection capability of protons enables to suppress systematic errors in electron spectrum due to uncertainties in proton rejection factor calculation caused by possible interaction model dependence. The CALET detector is the most suitable detector to directly measure total electron spectrum up to $20 \mathrm{TeV}$.

\subsection{Trigger System}

Since $\mathrm{GeV}$ cosmic rays are dominant, and it takes about $5 \mathrm{~ms}$ to be ready for the next event after building an event by retrieving all the ADC data from the detector components, it is necessary for CALET to select only higher energy particles by means of its trigger system. The trigger decision in CALET is based on the coincidence of trigger counter signals which are generated by discrimination of the analog signals from the detector components, i.e., each of CHD X and Y, IMC $\mathrm{X} 1-\mathrm{X} 4, \mathrm{Y} 1-\mathrm{Y} 4$, and TASC X1 generates lower discriminator (LD) signals. Because two IMC fiber layers are readout in one front end circuit, the number of trigger counter signals are reduced to 4 in each axis. TASC X1 is readout by photomultipliers to retrieve fast trigger signals although other 11 layers are readout by PD/APD to ensure wide dynamic range of 6 orders of magnitude.To take data under different conditions efficiently, CALET features three trigger modes as follows:

High Energy Shower Trigger (HE): This is the main trigger mode for CALET because it targets high energy electrons of $10 \mathrm{GeV} \sim 20 \mathrm{TeV}$, high energy gamma-rays of $10 \mathrm{GeV} \sim 10 \mathrm{TeV}$, and protons and nuclei of a few $10 \mathrm{GeV} \sim 1000 \mathrm{TeV}$. By requiring a large energy deposit in the middle of the detector, high energy shower events are selectively triggered. As a result, it is possible to maximize exposure to the target particles while strongly suppressing low energy particles and achieving a large geometrical factor at the same time.

Low Energy Shower Trigger (LE): This is the same as the high energy shower trigger but it targets lower energy particles generating a shower in the detector. The primary targets of the 
LE trigger are low energy electrons of $1 \mathrm{GeV} \sim 10 \mathrm{GeV}$ at high latitude and GRB gamma rays of $\geq 1 \mathrm{GeV}$. To trigger electrons at high latitude, LD signals are required at CHD and IMC upper layers to restrict the incident angle of the particles. The energy deposit required in the middle of the detector is much lower than for the HE trigger, but it is significantly higher than that of a minimum ionizing particle (MIP).

Single Trigger (Single): This trigger mode is dedicated to taking data of non-interacting particles for the detector calibration. It requires the energy deposit of a MIP and corresponding LDsignals in all of CHD, IMC and the upmost layer of TASC.

Since the combination of LDs required to generate the trigger signal is freely selectable, a new trigger mode such as the one dedicated to ultra heavy nuclei [ [ $]$ is also possible. The trigger decision is done by logical OR of the trigger signals from all the trigger modes.

\subsection{Data Acquisition System}

The acquisition of cosmic-ray event data is carried out by Mission Data Controller (MDC). Event data acquisition consists of event building task, event processing task, and event delivering task. Since the tasks use data buffer to communicate with each other, it is possible to parallelize the tasks. Because of this design, MDC can handle the sudden data increase, and observation dead time is minimized to the duration of event building task, maximizing the observation live time. Data downlink is controlled by the event delivering task and it limits the bandwidth usage up to $600 \mathrm{kbps}$ at maximum. To effectively compress the event data which exceeds 8000 channels, zero-suppression of the obtained ADC data is carried out in the event processing task.

In addition, MDC takes care of control of CALET detector components, CALET Gamma-ray Burst Monitor (CGBM) and support sensors. Support sensors include Advanced Stellar Compass which determines attitude of the CALET, GPS receiver which synchronizes MDC time to GPS time, and HV-Box which supplies high voltage to detectors. MDC also takes care of data collection of those detectors and equipment. In the ever-changing cosmic-ray radiation environment at the ISS orbit due to the influence of geomagnetism, it is necessary to control trigger rate and data amount by applying appropriate trigger condition and adjusting zero-suppression thresholds. It is one of the most important role of WCOC to plan such scientific observation to obtain fruitful data effectively.

\section{Simulation of the CALET Telemetry Data in ISS Environment}

To develop the WCOC system and to perform appropriate scientific observation on orbit, it is critically important to prepare simulation system to predict the CALET data onboard ISS with high accuracy. For this purpose, we have developed the CALET telemetry data simulation system based on the precedent development [B] for detector calibration onboard ISS. In the newly developed system, it is possible to simulate CALET telemetry data with high fidelity for any observation setting. The specific procedure to generate simulated CALET telemetry data is as follows:

1. Cosmic-ray flux calculation on ISS orbit: By using primary cosmic-ray spectra of protons, helium nuclei, electrons and positrons as input, ATMNC3 [䧃] calculates the transport of cosmic-rays in geomagnetism and atmosphere. All nuclei heavier than helium are counted as helium. Individual particles are repeatedly sampled and traced on a spherical shell at an altitude of $400 \mathrm{~km}$ to simulate ISS orbits. The list of latitude, longitude, particle species, 
energy, incident zenith and azimuthal angles is created. Then, the list is sorted to equi-solidangle grids [5] in geographic coordinate system and stored as particle library at ISS altitude.

2. Sampling cosmic-rays along ISS orbit: The cosmic rays incident on the CALET detector are sampled by utilizing location information of ISS and corresponding cosmic-ray list. First, the time until the next cosmic-ray incidence is randomly sampled from exponential distribution where the cosmic-ray rate is taken from the corresponding list. Then, the particle characteristic is sampled from the list in which solid angle correction is applied to simulate the incidence to the 3-dimensional CALET detector while the cosmic-ray list is created for the 2-dimensional spherical shell. At this point, the particles are sampled under the condition that they cross the sphere which contains the whole CALET model.

3. Detector simulation using EPICS: Sampled particles are then injected to CALET CAD model to calculate energy deposit $(\Delta E)$ at each scintillators by simulating the particle interactions inside the detector. As a direction of incident particle, zenith and azimuthal angles taken from the particle library are used. However, the incident position is randomly sampled here. By assuming that the cosmic-ray flux is homogeneous in each equi-solid-angle grid, the statistics of the cosmic-ray list can be used effectively. The CALET CAD model is the CALET simulation model based on the CALET mechanical drawing. It imports detailed information of supporting structures and other materials. We used EPICS ver.9.165 [目] as a simulation code where dpmjet-3 [ $[\mathbb{]}]$ is used as the hadronic interaction model.

4. MDC simulation: In order to leverage the telemetry data simulation system to plan scientific observation as well as to develop WCOC software for real-time monitoring and science data processing, it is a must to simulate the functions of MDC responsible for control and data acquisition of CALET in detail. For this purpose, we have developed "MDC Simulator" in which observation setting such as trigger and zero-suppression threshold can be specified. In the MDC simulator, (1) dead time treatment, (2) buffering function, (3) data delivery process, (4) digitization of $\Delta E$ at sensitive scintillators and (5) zero-suppression process are simulated faithfully. As a result, it is possible to simulate in detail the CALET telemetry data in each and every ISS environment and trigger conditions.

5. Telemetry data output: The data processed in the MDC simulator are output in a binary format which simulates the packets used in a real operation. The output data include periodically available housekeeping data as well as cosmic-ray event data. Since the MDC simulator simulates the output timing of each packets, the system is used to validate and to measure limit performance of CALET-GSE at JAXA in addition to the WCOC development.

Using the completed telemetry data simulation system, one ISS orbit data is generated in nominal observation condition as shown in the left panel of Fig. []. By analyzing the data, trend of quantities important to monitor the observation status is plotted in the right panel of Fig. [3. HE trigger rate (red line) and DAQ rate (black line) varies in the range of $10 \sim 25 \mathrm{~Hz}$ due mainly to the effect of rigidity cutoff. Dead time fraction (pink line) varies around $10 \%$. Because of dead time, DAQ rate always takes a bit smaller value than HE trigger rate. While taking the HE triggered cosmic-ray shower events, data transmission rate (blue line) varies in the range of 250 450 kbps, demonstrating that all the data can be downlinked within the maximum data rate of 600kbps. On the other hand, pedestal data are taken for two seconds twice in one orbit. Since zero-suppression 
is not applied to pedestal data, the data transmission rate immediately reaches its maximum value. While the usage of 1 st buffer (orange line) is always $0 \%$, 2nd buffer usage (green line; denoted as med. buffer) reaches $60 \%$ when taking pedestal data and decreases gradually after that. The data exceeding transmission limit are buffered and transmitted gradually. Owing to the buffering capability of MDC, data acquisition with HE trigger mode was restarted immediately after the pedestal data acquisition. Because it is possible to produce such a simulated telemetry data in any operation conditions, telemetry data simulation system plays a critical role in all of development of real-time monitoring system, scientific data processing, and operation planing.
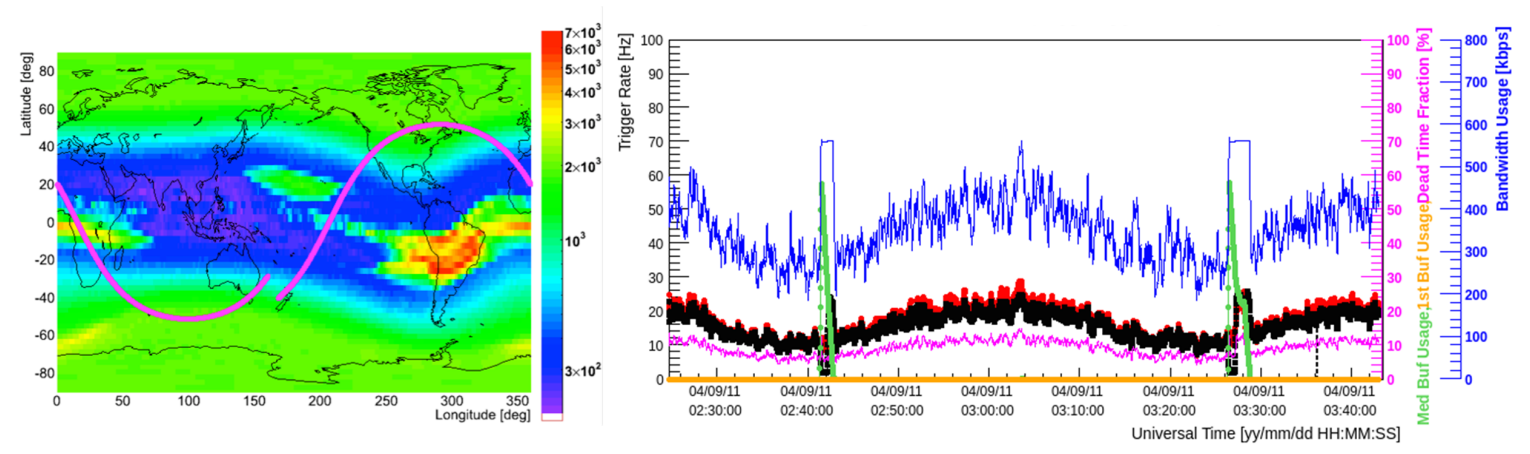

Figure 3: Example of simulated CALET telemetry data in ISS environment. (Left) simulated orbit of CALET onboard ISS in the cosmic ray intensity map at the ISS altitude calculated by ATMNC3, (Right) Typical telemetry data for one ISS orbit in nominal operation mode where only the HE trigger is enabled.

\section{Waseda CALET Operations Center}

\subsection{Real-time Monitoring}

To monitor the observation status of CALET in real-time, a quick look (QL) system which consolidates and visualizes cosmic-ray event data and housekeeping data was developed. The left panel of Fig. (1) summarizes the QL system. The telemetry data consists of 8164 channels of detector readout and more than 320 items of housekeeping data including $134 \mathrm{ch}$ of high voltage power supply, $80 \mathrm{ch}$ of temperature sensors, etc. Since large amount of data must be monitored in realtime in a comprehensive manner, it is necessary to summarize the data and to detect malfunctions automatically. The QL system provides such functions. For example, summary display summarizes information for operators to monitor on a steady basis and the QL program itself comprehensively judges the operation status. It issues an alert with color and sound when needed. Event display visualizes the cosmic-ray event in real time, making it possible to get intuitive understanding of the detector status including soundness check of each sensor. The ISS orbit monitor confirms the operation planning based on orbit prediction. Trend monitors visualize the temporal variation of observation data, playing an important role in finding the cause of issues and/or predicting future problems. On the other hand, the current data monitor displays the data comprehensively at any given instant. It is capable of displaying raw values as necessary. Including the histogram monitor, the QL system enables the detailed monitoring of the CALET status. By utilizing simulated CALET telemetry data, the QL systems are developed early on in a simulated situation of on-orbit operation, enabling the QL system to be used in CALET system tests. It already has past results of 
real-time monitoring. In a steady operation phase, the operator at WCOC will make excellent use of the QL system and will monitor the CALET all day and night.

\subsection{Science Data Processing}

The analysis scheme of CALET is shown in the right panel of Fig. 因. The scientific raw data called CALET Level-0 data are generated in JAXA CALET-GSE and transferred to WCOC. In the Level-0 data, although the time order is corrected and missing data are complemented, it is a simple format where received packets are only connected. In WCOC we convert them into Level-1 data which is the base data for scientific data analysis and distribute them to international CALET collaboration for scientific analysis. Quick analyses based on both Level-0 and Level-1 data are performed and their results are used as feedback for better operation planning and realtime monitoring. Various detector calibration will be performed using the CALET Level-1 data and Level-2 data for physics analysis will be produced. Spectra data and subset of data for individual science target will be called as Level-3 data or higher [ [8]. The data analysis using each data set will be carried out independently in each institute, and the official dataset to be used for publication will be processed at WCOC. WCOC takes an important part in CALET data analysis [Q]. The CALET Level-1 format is now fixed and the software development for flight data analysis and detector calibration using ground data are in progress using the CALET Level-1 data.
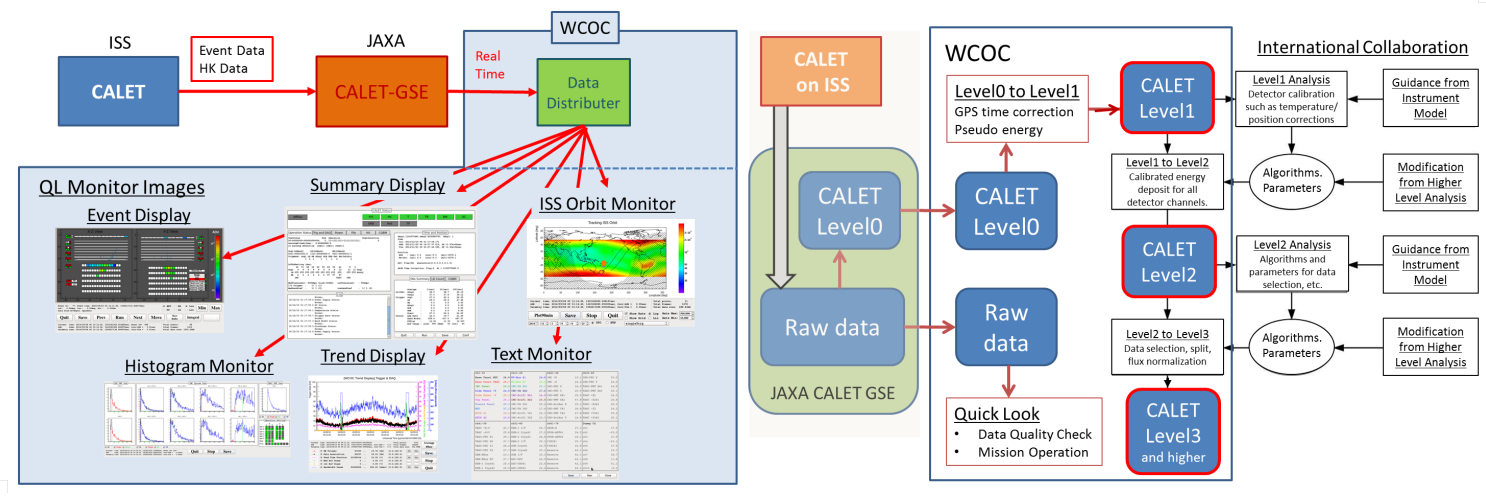

Figure 4: WCOC QL system (left) and CALET analysis procedure (right).

\subsection{Science Operation Planning}

The scientific operation of CALET will be performed using scheduled command file in which time stamped command sequence is described. While the most important task in steady operation is to continuously take high energy shower trigger data to precisely determine electron spectrum in $\mathrm{TeV}$ region, it is also necessary to take calibration data such as pedestal and penetrating particle data to calibrate MIP signals using single trigger mode. In addition, $\mathrm{GeV}$ gamma-ray observations synchronized to GRB trigger issued in CGBM detector, low energy electron data taking at high latitude, and other physics targets are also interesting as long as they do not affect the primary physics target of CALET. It is important to maximize the science outcome of CALET by means of efficient observations well planned in advance. For this purpose it is necessary to predict the relation between energy threshold and trigger rate. The left panel of Fig. \$ shows the triggerefficiency dependence on LD threshold. The higher the LD threshold goes, so does the energy 
threshold which is defined as the energy corresponding to a 50\% efficiency. The trigger efficiency was calculated for isotropic electron incidence while imposing the geometric condition [ए0]. From the on-orbit telemetry simulation data, trigger rate maps for various LD threshold settings were obtained. Each data acquisition costs $5 \mathrm{msec}$ of dead time. Since most of the triggers are due to protons and heliums, the associated dead time leads to a loss of live time for observing electrons. The right panel of Fig. [ shows the dependence of a trigger rate on energy threshold. Since trigger rate strongly depends on rigidity cutoff, the dependences are shown for several geomagnetic latitude range in different colors. Such kind of data are basic data to plan scientific operations. Based on the predictions for each operation mode at ISS environment and estimation of trigger rate dependence on ISS position, fine tuning of observation condition is in progress.
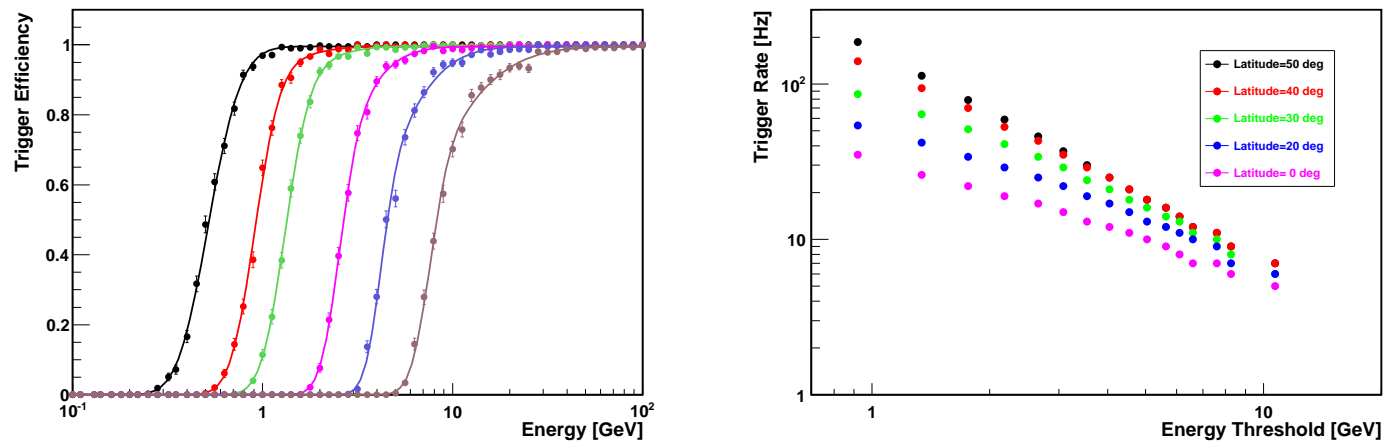

Figure 5: (Left) Trigger efficiency for electrons at different LD threshold settings, where an energy threshold is defined as the energy corresponding to the 50\% efficiency. (Right) the dependence of trigger rate on energy threshold. Geomagnetic latitude dependence is also shown as different colors, i.e., black, red, green, blue and magenta dots denotes $\sim 50 \mathrm{deg}, \sim 40 \mathrm{deg}, \sim 30 \mathrm{deg}, \sim 20 \mathrm{deg}, \sim 0 \mathrm{deg}$, respectively.

\section{Conclusion}

The HTV5 which will carry CALET to ISS will be launched on August 2015. WCOC has three important roles to perform scientific operations of CALET, namely operation planning, real-time monitoring, and scientific data processing. WCOC is ready to receive data and is waiting for the first cosmic-ray event of CALET onboard ISS.

This work was supported by JSPS KAKENHI Grant Number 26220708.

\section{References}

[1] S. Torii et al., Proc. of the 34th ICRC (The Hague, Netherlands), this conference (2015).

[2] B. F. Rauch et al., Proc. 32nd ICRC (Beijing, China), Vol.6, (2011) 355.

[3] T. Niita et al., Adv. Space Res. 55 (2015) 2500-2508.

[4] M. Honda et al., PRD 70 (2004) 043008.

[5] I.I. Gringorten and P.J. Yepez, Instrumentation Papers No.343 (1992).

[6] "EPICS Home Page" http://cosmos.n.kanagawa-u.ac.jp/EPICSHome/

[7] "DPMJET3 Home Page" http://sroesler.web.cern.ch/sroesler/dpmjet3.html

[8] T.G. Guzik et al., Proc. of 33rd ICRC (Rio de Janeiro, Brazil), 168 (2013).

[9] S. Torii, J.P. Wefel, P.S. Marrocchesi, Data Processing and Analysis Plan for the CALorimetric Electron Telescope (CALET) on the International Space Station, JAXA, Rev. B, August 2014.

[10] Y. Akaike et al., Proc. of the 32nd ICRC (Beijing, China), Vol.6, (2011) 371. 